\title{
Bosonization in three spatial dimensions and a 2-form gauge theory
}

\author{
Yu-An Chen $\odot^{*}$ and Anton Kapustin \\ California Institute of Technology, Pasadena, California 91125, USA
}

(Received 7 September 2019; revised manuscript received 21 November 2019; published 16 December 2019)

\begin{abstract}
We describe a $3 \mathrm{~d}$ analog of the Jordan-Wigner transformation which maps an arbitrary fermionic system on a $3 \mathrm{~d}$ spatial lattice to a 2 -form $\mathbb{Z}_{2}$ gauge theory with an unusual Gauss law. An important property of this map is that it preserves the locality of the Hamiltonian. The map depends explicitly on the choice of a spin structure of the spatial manifold. We give examples of $3 \mathrm{~d}$ bosonic systems dual to free fermions. We also describe the corresponding Euclidean lattice models, which is analogous to the Steenrod square term in $(3+1) \mathrm{D}$ [compared to the Chern-Simon term in $(2+1) \mathrm{D}]$.
\end{abstract}

DOI: $10.1103 /$ PhysRevB.100.245127

\section{INTRODUCTION AND SUMMARY}

It is well known that every lattice fermionic system in $1 \mathrm{~d}$ is dual to a lattice system of spins with a $\mathbb{Z}_{2}$ global symmetry (and vice versa). The duality is kinematic (independent of a particular Hamiltonian) and arises from the Jordan-Wigner transformation. Recently it has been shown that any lattice fermionic system in $2 \mathrm{~d}$ is dual to a $\mathbb{Z}_{2}$ gauge theory with an unusual Gauss law [1]. The fermion can be identified with the flux excitation of the gauge theory. The $2 \mathrm{~d}$ duality is also kinematic. In this paper we extend these results to $3 \mathrm{~d}$ systems. We show that every lattice fermionic system in $3 \mathrm{~d}$ is dual to a $\mathbb{Z}_{2}$ 2-form gauge theory with an unusual Gauss law. Here "2-form gauge theory" means that the $\mathbb{Z}_{2}$ variables live on plaquettes, while the parameters of the gauge symmetry live on links. 2-form gauge theories in 3+1D have local flux excitations, and the unusual Gauss law ensures that these excitations are fermions [2].

The form of the modified Gauss law is largely dictated by the observation first made in Ref. [3] that a bosonization of fermionic systems in $d$ dimensions must have a global $(d-1)$-form $\mathbb{Z}_{2}$ symmetry with a particular't Hooft anomaly. The standard Gauss law leads to a trivial 't Hooft anomaly, so bosonization requires us to modify it in a particular way. The precise form of the modified Gauss law and the bosonization map depends on the choice of the lattice. We describe them in two cases: the cubic lattice and a $3 \mathrm{~d}$ triangulation.

Our $3 \mathrm{~d}$ bosonization map is kinematic and local in the same sense as the Jordan-Wigner map: Every local bosonic observable on the fermionic side, including the Hamiltonian density, is mapped to a local gauge-invariant observable on the gauge theory side. In particular, the product of any two fermions is mapped to a local observable on the gauge theory side. A single fermion is not mapped to a local observable. Rather, it is mapped to an observable supported on a string which runs off to infinity. This is also analogous to the usual 1d Jordan-Wigner transformation.

*ychen2@caltech.edu
In the literature, there are examples of specific bosonic models in $3 \mathrm{~d}$ with emergent fermions. Our general construction is reminiscent of the work by Levin and Wen [4]. These authors constructed systems of rotors which have emergent fermions. In our approach rotors are replaced with $\mathbb{Z}_{2}$ spins. There are also several proposals for an analog of the JordanWigner map in 2d or arbitrary dimensions [5-8]. Our construction is most similar to that of Bravyi and Kitaev [6]. One advantage of our construction is that we can clearly identify the kind of $3 \mathrm{~d}$ bosonic systems that are dual to fermionic systems: They possess global 2-form $\mathbb{Z}_{2}$ symmetry with a specific 't Hooft anomaly, as proposed in Ref. [3]. It is also manifest in our approach that the bosonization map depends on a choice of spin structure.

Our bosonization method allows for an easy construction of bosonic systems dual to free fermions with an arbitrary dispersion law. As an illustration, we describe a bosonic model on a cubic lattice whose dual fermionic description involves Dirac cones. It can be regarded as a $3 \mathrm{~d}$ analog of the Kitaev honeycomb model. Other $3 \mathrm{~d}$ analogs of the honeycomb model have been proposed in Refs. $[9,10]$. We also identify some Euclidean bosonic 4D models which are dual to free fermions. These models can be understood as 2 -form $\mathbb{Z}_{2}$ gauge theories whose action involves a topological term.

The content of the paper is as follows. In Sec. II we describe our $3 \mathrm{~d}$ bosonization map, first on a cubic lattice and then on a $3 d$ triangulation. In Sec. III we describe special instances of the $3 \mathrm{~d}$ bosonization map and give a Euclidean lattice formulation of some 2-form $3 \mathrm{~d}$ gauge theories dual to free fermions. In Sec. IV we construct 3d bosonic lattice models dual to free fermions with gauged fermion parity. Unlike in previous examples, the Hilbert space on the bosonic side is unconstrained. In particular, we construct a $3 \mathrm{~d}$ model of unconstrained bosonic spins on a cubic lattice which is dual to a theory of free Dirac cones. Section V summarizes our results. In Appendix A we review some mathematical notions used in the paper (chains, cochains, cup product, and Steenrod's higher cup product). In Appendix B we review $\mathbb{Z}_{2}$ lattice gauge theories and compare ordinary and modified $\mathbb{Z}_{2}$ gauge theories. 


\section{BOSONIZATION ON A THREE-DIMENSIONAI LATTICE}

\section{A. Cubic lattice}

We begin by reviewing the $2 \mathrm{~d}$ bosonization on a square lattice following Ref. [1]. The elements of vertices, edges, and faces are denoted $v, e, f$. On each face $f$ of the lattice we place a single pair of fermionic creation-annihilation operators $c_{f}, c_{f}^{\dagger}$, or equivalently a pair of Majorana fermions $\gamma_{f}, \gamma_{f}^{\prime}$. The even fermionic algebra consists of local observables with a trivial fermionic parity (i.e., those local observables which commute with the total fermion parity $\left.(-1)^{F} \equiv \prod_{f}(-1)^{c_{f}^{\dagger} c_{f}}\right)$. The even algebra is generated by

$$
(-1)^{F_{f}}=-i \gamma_{f} \gamma_{f}^{\prime}
$$

and

$$
S_{e}=i \gamma_{L(e)} \gamma_{R(e)}^{\prime}
$$

where $L(e)$ and $R(e)$ are faces to the left and right of $e$, with respect to some orientation of $e$.

Note that the even fermionic algebra (which is the same as the algebra of local observables containing an even number of Majorana operators) is generated by $(-1)^{F_{f}}$ and $S_{e}$. That is, any such local observable can be written as a linear combination of products of operators $(-1)^{F_{f}}$ and $S_{e}$. In particular, this applies to any fermion bilinear involving two Majorana fermions on any two faces and to interaction terms in an arbitrary finite-range Hamiltonian. For example, since $\gamma_{L(e)} \gamma_{R(e)}=-S_{e}(-1)^{F_{R(e)}}$, a product of Majorana fermions living on adjacent faces can be so generated. For $\gamma_{f_{i}} \gamma_{f_{j}}$ where $f_{i}$ and $f_{j}$ are not adjacent, we find a path $l=f_{i}, f_{1}, f_{2}, \ldots, f_{n}, f_{j}$ from $f_{i}$ to $f_{j}$ and express $\gamma_{f_{i}} \gamma_{f_{j}}$ as $\left(\gamma_{f_{i}} \gamma_{f_{1}}\right)\left(\gamma_{f_{1}} \gamma_{f_{2}}\right) \cdots\left(\gamma_{f_{n-1}} \gamma_{f_{n}}\right)\left(\gamma_{f_{n}} \gamma_{f_{j}}\right)$. Each adjacent pair of Majorana operator is a product of $(-1)^{F_{f}}$ and $S_{e}$, so $\gamma_{f_{i}} \gamma_{f_{j}}$ is also generated. For interaction terms, such as $\gamma_{f_{i}} \gamma_{f_{j}} \gamma_{f_{k}} \gamma_{f_{l}}$ for arbitrary faces $f_{i}, f_{j}, f_{k}, f_{l}$, we pair up Majorana fermions as $\gamma_{f_{i}} \gamma_{f_{j}}$ and $\gamma_{f_{k}} \gamma_{f_{l}}$ and write each of them as a product of some $(-1)^{F_{f}}$ and $S_{e}$. Therefore all even fermionic operators can be written as linear combinations of products of $(-1)^{F_{f}}$ and $S_{e}$.

The bosonic dual of this system involves $\mathbb{Z}_{2}$-valued spins on the edges of the square lattice. For every edge $e$ we define a unitary operator $U_{e}$ which squares to 1 . Labeling the faces and vertices as in Fig. 1, we define:

$$
\begin{aligned}
& U_{56}=X_{56} Z_{25} \\
& U_{58}=X_{58} Z_{45}
\end{aligned}
$$

where $X, Z$ are Pauli matrices acting on a spin at each edge:

$$
X_{e}=\left[\begin{array}{ll}
0 & 1 \\
1 & 0
\end{array}\right], \quad Z_{e}=\left[\begin{array}{rr}
1 & 0 \\
0 & -1
\end{array}\right] .
$$

Operators $U_{e}$ for other edges are defined by using translation symmetry.

It has been shown in Ref. [1] that $U_{e}$ and $S_{e}$ satisfy the same commutation relations. We also identify fermionic parity $(-1)^{F_{f}}$ at each face with the "flux operator" $W_{f} \equiv \prod_{e \subset f} Z_{e}$. The bosonization map is

$$
\begin{aligned}
(-1)^{F_{f}} & =-i \gamma_{f} \gamma_{f}^{\prime} \longleftrightarrow W_{f} \\
S_{e} & =i \gamma_{L(e)} \gamma_{R(e)}^{\prime} \longleftrightarrow U_{e} .
\end{aligned}
$$

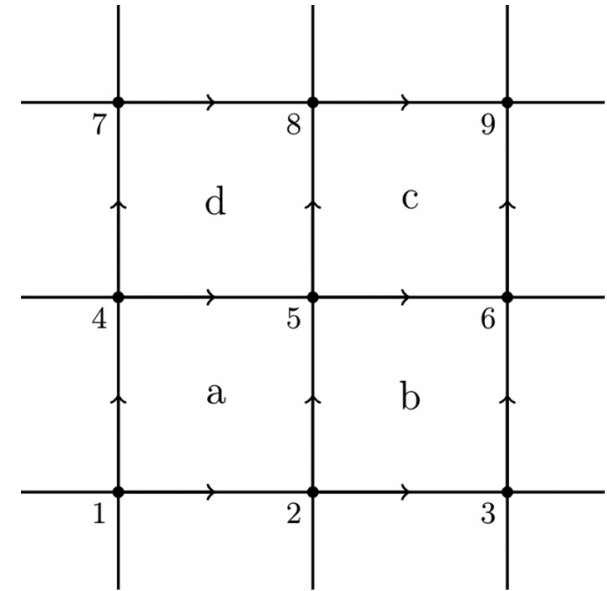

FIG. 1. Bosonization on a square lattice requires constraints on vertices.

The condition $(-1)^{F_{a}}(-1)^{F_{c}} S_{58} S_{56} S_{25} S_{45}=1$ on fermionic operators gives gauge constraints $G_{v}=W_{f_{c}} \prod_{e \supset v_{5}} X_{e}=1$ for bosonic operators, or generally

$$
W_{\mathrm{NE}(v)} \prod_{e \supset v} X_{e}=1
$$

where $\operatorname{NE}(v)$ is the face northeast of $v$. Equation (6) is the modified Gauss law (gauge constraint) and the bosonic theory is a $2 \mathrm{~d} \mathbb{Z}_{2}$ gauge theory, Hilbert space generated by $\mathbb{Z}_{2}$ spins (Pauli matrices) subjected to gauge constraints $G_{v}=1$. For comparison, the standard $2 \mathrm{~d} \mathbb{Z}_{2}$ gauge theory has the same variables, but the Gauss law is $\prod_{e \supset v} X_{e}=1$. The standard $\mathbb{Z}_{2}$ gauge theory is dual to a model of spins by $2 \mathrm{~d}$ KramersWannier duality, while the modified $\mathbb{Z}_{2}$ gauge theory is dual to a model of fermions by our construction. These equivalences are kinematic, in the sense that they work for any finite-range Hamiltonian.

Next, we introduce our bosonization method on an infinite $3 \mathrm{~d}$ cubic lattice. Suppose that we have a model with fermions living at the centers of cubes. Let us describe the generators and relations in the algebra of local observables with trivial fermion parity (the even fermionic algebra for short).

On each cube $t$ we have a single fermionic creation operator $c_{t}$ and a single fermionic annihilation operator $c_{t}^{\dagger}$ with the usual anticommutation relations. The fermionic parity operator on cube $t$ is $(-1)^{F_{t}}=(-1)^{c_{t}^{\dagger} c_{t}}$. It is a " $\mathbb{Z}_{2}$ operator" (i.e., it squares to 1$)$. All operators $(-1)^{F_{t}}$ commute with each other. The even fermionic algebra is generated by these operators and the operators $c_{t}^{\dagger} c_{t^{\prime}}, c_{t} c_{t^{\prime}}$, and their Hermitean conjugates, where $t$ and $t^{\prime}$ are two cubes which share a face. Overall, we get four generators for each face and one generator for each cube. It is easier to work in the Majorana basis

$$
\gamma_{t}=c_{t}+c_{t}^{\dagger}, \quad \gamma_{t}^{\prime}=\left(c_{t}-c_{t}^{\dagger}\right) / i .
$$

The even fermionic algebra is generated by $i \gamma_{L(f)} \gamma_{R(f)}^{\prime}$ and $-i \gamma_{t} \gamma_{t}^{\prime}$ where each face is assigned an orientation from cube $L(f)$ to cube $R(f)$.

To illustrate the definition of these operators, we draw the dual lattice of the original lattice. In Fig. 2, fermions live on vertices and the orientations of each dual edge (face 


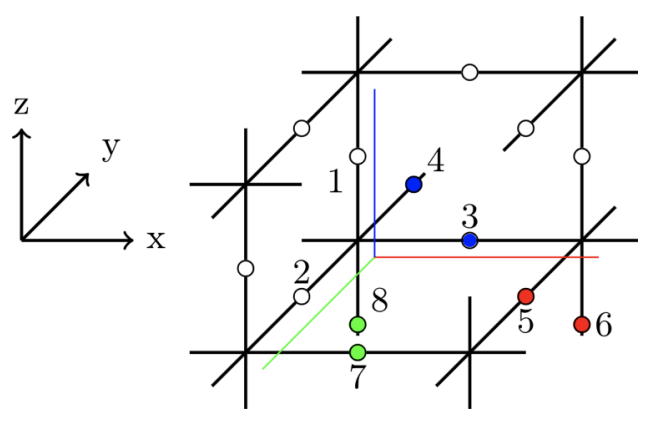

FIG. 2. For edges in the dual lattice, the "framing" is defined by green, red, and blue edges, which is a small shift of duel edges [4]. Given a dual edge $f$, the operator $U_{f}$ is defined as $X_{f}$ times $Z_{f^{\prime}}$ for those $f^{\prime}$ which intersect the framing of $f$ when projected to the plane (i.e., $U_{f_{1}}=X_{1} Z_{3} Z_{4}, U_{f_{2}}=X_{2} Z_{7} Z_{8}$, and $U_{f_{3}}=X_{3} Z_{5} Z_{6}$ ).

of the original lattice) are taken to be along $+x,+y$, and $+z$ directions. The Majorana hopping operator is defined by $S_{f}=i \gamma_{L(f)} \gamma_{R(f)}^{\prime}$ where $L(f)$ and $R(f)$ are source and sink (starting and ending points) of dual edge $f$ in the dual lattice. $S_{f_{i}}$ and $S_{f_{j}}$ anticommute only when both dual edges $f_{i}$ and $f_{j}$ start from the same point or both end at the same point.

The dual bosonic system has $\mathbb{Z}_{2}$ spins living on faces of the original lattice (or equivalently, on edges of the dual lattice). To define bosonic hopping operators $U_{f}$, we need to choose a framing for each edge of the dual lattice, i.e., a small shift of each dual edge along some orthogonal direction. We also assume that when projected on some generic plane (such as the plane of the page) a shifted dual edge intersects all dual edges transversally. For example, in Fig. 2 such a framing is indicated by red, green, and blue lines (for dual edges along $x$, $y$, and $z$ directions, respectively), and the shift of the dual edge 1 intersects dual edges 3 and 4 [11]. Now we define $U_{f}$ as a product of $X_{f}$ with all $Z_{f^{\prime}}$ such that $f^{\prime}$ intersects the framing of $f$ when projected to the plane of the page. For example, the hopping operator for the dual edge 1 is $U_{1}=X_{1} Z_{3} Z_{4}$. Notice that $U_{1}, U_{3}$, and $U_{4}$ anticommute with each other and $U_{3}$, $U_{5}$, and $U_{6}$ anticommute with each other, while $U_{2}$ and $U_{3}$ commute, and $U_{1}$ and $U_{8}$ commute.

One can check that $S_{f}$ and $U_{f}$ have the same commutation relations. Therefore, the bosonization map in $3 \mathrm{D}$ can be defined as follows:

(1) For any cube $t$ let $W_{t} \equiv \prod_{f \subset t} Z_{f}$. We identify the fermionic states $\left|F_{t}=0\right\rangle$ and $\left|F_{t}=1\right\rangle$ with bosonic states for which $W_{t}=1$ and $W_{t}=-1$, respectively. Thus

$$
(-1)^{F_{t}}=-i \gamma_{t} \gamma_{t}^{\prime} \longleftrightarrow W_{t} .
$$

(2) The fermionic hopping operator $S_{f}$ is identified with $U_{f}$ defined above:

$$
S_{f}=i \gamma_{L(f)} \gamma_{R(f)}^{\prime} \longleftrightarrow U_{f} .
$$

As in $2 \mathrm{~d}$, the bosonic operators satisfy some constraints. In Fig. 3, we calculate the product of $S_{f}$ around the red square on the dual lattice:

$$
\begin{aligned}
S_{f_{1}} S_{f_{2}} S_{f_{3}} S_{f_{4}} & =\left(i \gamma_{d} \gamma_{c}^{\prime}\right)\left(i \gamma_{b} \gamma_{c}^{\prime}\right)\left(i \gamma_{a} \gamma_{b}^{\prime}\right)\left(i \gamma_{a} \gamma_{d}^{\prime}\right) \\
& =-\left(-i \gamma_{b} \gamma_{b}^{\prime}\right)\left(-i \gamma_{d} \gamma_{d}^{\prime}\right) \\
& =-(-1)^{F_{b}}(-1)^{F_{d}} \longleftrightarrow-W_{b} W_{d} .
\end{aligned}
$$

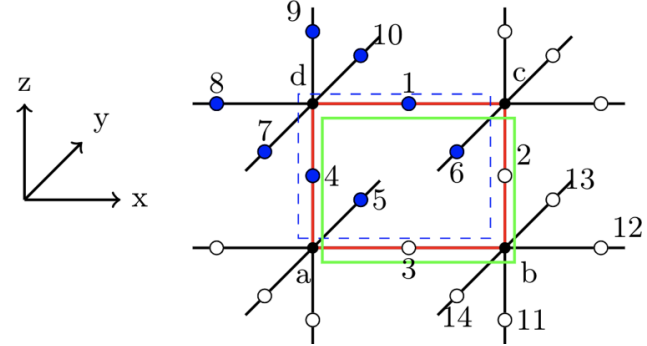

FIG. 3. The framing of the hopping term defined previously is indicated by the green square, while the gauge constraint involves the $Z$ operators in the opposite framing (blue dashed square).

Its bosonic dual defined by (9) is the product of the corresponding operators $U_{f}$. Their definition involves a framing of the red square given by the green square:

$$
\begin{aligned}
U_{f_{1}} U_{f_{2}} U_{f_{3}} U_{f_{4}} & =\left(X_{1} Z_{2} Z_{6}\right)\left(X_{2} Z_{12} Z_{13}\right)\left(X_{3} Z_{11} Z_{14}\right)\left(X_{4} Z_{3} Z_{5}\right) \\
& =-X_{1} X_{2} X_{3} X_{4} Z_{5} Z_{6}\left(Z_{2} Z_{3} Z_{11} Z_{12} Z_{13} Z_{14}\right) \\
& =-X_{1} X_{2} X_{3} X_{4} Z_{5} Z_{6} W_{b}
\end{aligned}
$$

Comparing (10) and (11), we get the constraint

$$
1=X_{1} X_{2} X_{3} X_{4} Z_{5} Z_{6} W_{d}=X_{1} X_{2} X_{3} X_{4} Z_{1} Z_{4} Z_{5} Z_{6} Z_{7} Z_{8} Z_{9} Z_{10} .
$$

The operators $Z$ 's are the edges crossed by dashed square in Fig. 3. The framing for gauge constraints is opposite to the framing used to define hopping operators. We have a gauge constraint for each face of dual lattice. In terms of the original lattice, there is one gauge constraint for each edge. All these constraints commute and thus define a $\mathbb{Z}_{2} 2$-form gauge theory with an unusual Gauss law.

\section{B. Examples}

\section{Soluble 3+1D lattice gauge theories}

The standard Gauss law for a 2-form $\mathbb{Z}_{2}$ gauge theory is $\prod_{f \supset e} X_{f}=1$. Such a bosonic gauge theory is dual to a theory of bosonic spins living on the vertices of the dual lattice. In particular, the quantum Ising model can be described by a $\mathbb{Z}_{2}$ 2 -form gauge theory with the Hamiltonian

$$
H_{\text {Ising }}=g^{2} \sum_{f} X_{f}+\frac{1}{g^{2}} \sum_{t} W_{t} .
$$

This model is not soluble.

If we impose the modified Gauss law (12) instead, the simplest analogous gauge-invariant Hamiltonian is

$$
H_{b}=g^{2} \sum_{f} U_{f}+\frac{1}{g^{2}} \sum_{t} W_{t} .
$$

The first and second term can be thought of as the kinetic and potential energies, respectively. This is dual to the fermionic Hamiltonian

$$
\begin{aligned}
H_{f}= & t \sum_{f}\left(c_{L(f)} c_{R(f)}-c_{L(f)}^{\dagger} c_{R(f)}^{\dagger}\right. \\
& \left.+c_{L(f)}^{\dagger} c_{R(f)}+c_{R(f)}^{\dagger} c_{L(f)}\right)+\mu \sum_{t} c_{t}^{\dagger} c_{t}
\end{aligned}
$$


where $t=g^{2}$ and $\mu=\frac{2}{g^{2}}$. The fermionic Hamiltonian is free and thus soluble. By Fourier transform $c_{\vec{x}}=\frac{1}{\sqrt{N}} \sum_{\vec{k}} e^{i \vec{k} \cdot \vec{x}} c_{\vec{k}}$, the fermionic Hamiltonian becomes

$$
H_{f}=\sum_{\vec{k}} \epsilon_{\vec{k}} c_{\vec{k}}^{\dagger} c_{\vec{k}}+\sum_{\vec{k}}\left(\Delta_{\vec{k}} c_{\vec{k}} c_{-\vec{k}}+\text { H.c. }\right)
$$

with $\epsilon_{\vec{k}}=\mu+2 t\left(\cos k_{x}+\cos k_{y}+\cos k_{z}\right) \quad$ and $\quad \Delta_{\vec{k}}=$ $t\left(e^{-i k_{x}}+e^{-i k_{y}}+e^{-i k_{z}}\right)$. The Hamiltonian (16) can be written in the Bogoliubov-de-Gennes (BdG) formalism as

$$
H_{f}=\frac{1}{2} \sum_{\vec{k}} \Psi_{\vec{k}}^{\dagger} H_{\mathrm{BDG}}(\vec{k}) \Psi_{\vec{k}}
$$

with

$$
H_{\mathrm{BDG}}(\vec{k})=\left[\begin{array}{cc}
\epsilon_{\vec{k}} & -\Delta_{\vec{k}}^{*} \\
-\Delta_{\vec{k}} & -\epsilon_{\vec{k}}
\end{array}\right], \quad \Psi_{\vec{k}}=\left[\begin{array}{c}
c_{\vec{k}} \\
c_{-\vec{k}}^{\dagger}
\end{array}\right] .
$$

The spectrum is

$$
\begin{aligned}
E^{2}= & t^{2}\left(3+2 \cos \left(k_{x}-k_{y}\right)+2 \cos \left(k_{x}-k_{z}\right)+2 \cos \left(k_{y}-k_{z}\right)\right) \\
& +\left[\mu+2 t\left(\cos k_{x}+\cos k_{y}+\cos k_{z}\right)\right]^{2} .
\end{aligned}
$$

Notice that for $\mu=0$ the gap closes for $\vec{k}=\left(q, q+\frac{2 \pi}{3}, q+\right.$ $\frac{4 \pi}{3}$ ) for arbitrary $q$.

\section{Bosonic model with Dirac cones}

Using the bosonization map (8) and (9), we can construct an equivalent bosonic model for any arbitrary fermionic model. For instance, Ref. [12] constructs a fermionic model on a cubic lattice with Dirac cones:

$$
H=-t \sum_{\vec{r}}\left(s_{x}(\vec{r}) c_{\vec{r}+\hat{x}}^{\dagger} c_{\vec{r}}+s_{y}(\vec{r}) c_{\vec{r}+\hat{y}}^{\dagger} c_{\vec{r}}+s_{z}(\vec{r}) c_{\vec{r}+\hat{z}}^{\dagger} c_{\vec{r}}+\text { H.c. }\right)
$$

with $s_{x}(\vec{r}), s_{y}(\vec{r})$, and $s_{z}(\vec{r})$ defined as

$$
\begin{aligned}
& s_{x}(i, j, k)=1 \\
& s_{y}(i, j, k)=(-1)^{i} \\
& s_{z}(i, j, k)=(-1)^{i+j} .
\end{aligned}
$$

It is a model with nearest neighbor hopping. The spectrum is

$$
E= \pm 2 t \sqrt{\cos ^{2} k_{x}+\cos ^{2} k_{y}+\cos ^{2} k_{z}}
$$

with two Dirac cones at $\vec{k}=(\pi / 2, \pi / 2, \pi / 2)$ and $\vec{k}=$ $(3 \pi / 2, \pi / 2, \pi / 2)$. Applying the bosonization map, the corresponding bosonic Hamiltonian is

$$
\begin{aligned}
H= & -\frac{t}{2} \sum_{f_{x}} s_{x}\left(L\left(f_{x}\right)\right) U_{f_{x}}\left(1-W_{L\left(f_{x}\right)} W_{R\left(f_{x}\right)}\right) \\
& -\frac{t}{2} \sum_{f_{y}} s_{y}\left(L\left(f_{y}\right)\right) U_{f_{y}}\left(1-W_{L\left(f_{y}\right)} W_{R\left(f_{y}\right)}\right) \\
& -\frac{t}{2} \sum_{f_{z}} s_{z}\left(L\left(f_{z}\right)\right) U_{f_{z}}\left(1-W_{L\left(f_{z}\right)} W_{R\left(f_{z}\right)}\right),
\end{aligned}
$$

where $f_{x}, f_{y}, f_{z}$ refer to faces normal to $x, y, z$ directions, with gauge constraints (12). On the bosonic side, it is very nontrivial to see that the model describes Dirac cones.

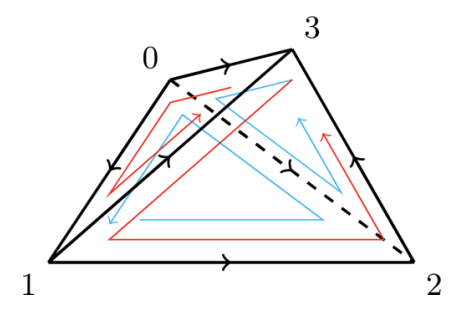

FIG. 4. A branching structure on a tetrahedron. The orientation of each face is determined by the right-hand rule. We defined this as the "+" tetrahedron, the directions of faces 012 and 023 are inward (blue) while the directions of faces 123 and 013 are outward (red). The directions of faces are reversed in the "-" tetrahedron (mirror image of this tetrahedron).

\section{Triangulation}

The notations in algebraic topology and conventions used in this letter are reviewed in Appendix A. The bosonization method described above also works for any triangulation. For an arbitrary triangulation $T$ of a $3 \mathrm{~d}$ manifold $M$, we choose a branching structure. A branching structure is a choice of an orientation on each edge such that there is no oriented loop on any triangle. One simple way is to label vertices by different numbers and assign the direction of an edge from the vertex with smaller number to the vertex with larger number (see Fig. 4). Each tetrahedron has two inward faces and two outward faces (by right-hand rule). We place fermions at the centers of tetrahedrons. Each tetrahedron $t$ contains Majorana operators $\gamma_{t}$ and $\gamma_{t}^{\prime}$. We define the fermionic hopping operator on each face $f$ as

$$
S_{f}=i \gamma_{L(f)} \gamma_{R(f)}^{\prime}
$$

where $L(f) / R(f)$ is the tetrahedron with $f$ as an outward/inward face. Notice that $S_{f}$ and $S_{f^{\prime}}$ anticommute only when $f$ and $f^{\prime}$ share a tetrahedron with both $f$ and $f^{\prime}$ inward or outward. To express this property mathematically, we introduce (higher) cup product used in algebraic topology. The definition and properties of the (higher) cup products are reviewed in Appendix A. If $\beta_{1}$ and $\beta_{2}$ are 2-cochains, then

$$
\beta_{1} \cup_{1} \beta_{2}(0123)=\beta_{1}(023) \beta_{2}(012)+\beta_{1}(013) \beta_{2}(123) .
$$

Therefore, the commutation relations can be expressed as

$$
S_{f} S_{f^{\prime}}=(-1)^{\int f \cup_{1} f^{\prime}+f^{\prime} \cup_{1} f} S_{f^{\prime}} S_{f}
$$

where we abuse the notation $f \in C^{2}\left(T, \mathbb{Z}_{2}\right)$ as a 2-cochain with value 1 on face $f$ and 0 otherwise. The even fermionic algebra is generated by the operators $S_{f}$ for all faces and the fermionic parity operators $(-1)^{F_{t}}$ for all tetrahedra.

The dual bosonic variables are $\mathbb{Z}_{2}$ spins which live on faces of the triangulation. As before, the flux operator

$$
W_{t}=\prod_{f \supset t} X_{f}
$$

corresponds to $(-1)^{F_{t}}$ under the bosonization map.

Next we need to find bosonic operators $U_{f}$ which have the same commutation relation as fermionic operators $S_{f}$. We should define $U_{f}$ as $X_{f}$ times $Z_{f^{\prime}}$ for some faces $f^{\prime}$ which share a tetrahedron with $f$ and have the same orientation with 
respect to the tetrahedron. One way to define $U_{f}$ is

$$
U_{f}=X_{f} \prod_{t \in\{L(f), R(f)\}} Z_{t_{023}}^{f\left(t_{012}\right)} Z_{t_{013}}^{f\left(t_{123}\right)}=X_{f} \prod_{f^{\prime}} Z_{f^{\prime}}^{\int f^{\prime} \cup_{1} f} .
$$

$U_{f}$ satisfy the commutation relation

$$
U_{f} U_{f^{\prime}}=(-1)^{\int f \cup_{1} f^{\prime}+f^{\prime} \cup_{1} f_{f^{\prime}}} U_{f}
$$

which is the same as (26).

The final step is to determine the constraints on bosonic variables. There is one such constraint for each edge $e$. In the product $\prod_{f \supset e} S_{f}$, the only surviving terms are $-i \gamma_{t} \gamma_{t}^{\prime}$ with one face going inward and one face going outward of $t$. The term $-i \gamma_{t} \gamma_{t}^{\prime}$ is bosonized to $W_{t} \equiv \prod_{f \subset t} Z_{f}$. Therefore, the product can be written as

$$
\prod_{f \supset e} S_{f} \sim \prod_{t \mid e=t_{01}, t_{03}, t_{12}, t_{23}} W_{t}
$$

where $\sim$ means that it is equal up to a sign, which will be treated carefully in the next paragraph. For a tetrahedron $t$ containing an edge $e$ with adjacent faces $f_{1}$ and $f_{2}$, consider the following product which gives $W_{t}$ for $e=t_{01}, t_{03}, t_{12}, t_{23}$ and 1 otherwise:

$$
\begin{array}{r}
Z_{f_{1}} Z_{f_{2}} \prod_{f^{\prime} \subset t} Z_{f^{\prime}}^{\left(f_{1}+f_{2}\right) \cup_{1} f^{\prime}+f^{\prime} \cup_{1}\left(f_{1}+f_{2}\right)} \\
= \begin{cases}W_{t}, & \text { if } e=t_{01}, t_{03}, t_{12}, t_{23} \\
1, & \text { otherwise }\end{cases}
\end{array}
$$

Substituting this into (29), we have

$$
\prod_{f \supset e} S_{f} \sim \prod_{f \supset e} \prod_{f^{\prime}} Z_{f^{\prime}}^{\int \boldsymbol{f}^{\prime} \cup_{1} \boldsymbol{f}+\boldsymbol{f} \cup_{1} \boldsymbol{f}^{\prime}}=\prod_{f^{\prime}} Z_{f^{\prime}}^{\int \boldsymbol{f}^{\prime} \cup_{1} \delta \boldsymbol{e}+\delta \boldsymbol{e} \cup_{1} \boldsymbol{f}^{\prime}} .
$$

On the other hand, the product $\prod_{f \supset e} U_{f}$ is

$$
\prod_{f \supset e} U_{f} \sim \prod_{f \supset e} X_{f} \prod_{f^{\prime}} Z_{f^{\prime}}^{\int \boldsymbol{f}^{\prime} \cup_{1} f}=\left(\prod_{f \supset e} X_{f}\right) \prod_{f^{\prime}} Z_{f^{\prime}}^{\int \boldsymbol{f}^{\prime} \cup_{1} \delta \boldsymbol{e}}
$$

Identifying (31) and (32) gives

$$
\left(\prod_{f \supset e} X_{f}\right) \prod_{f^{\prime}} Z_{f^{\prime}}^{\int \delta e \cup_{1} f^{\prime}}=1
$$

This is the modified Gauss law (gauge constraint) on each edge $e$. One can check that constraints for different edges $e_{1}$ and $e_{2}$ commute since

$$
\begin{aligned}
\int & \left(\delta \boldsymbol{e}_{1} \cup_{1} \delta \boldsymbol{e}_{2}+\delta \boldsymbol{e}_{2} \cup_{1} \delta \boldsymbol{e}_{1}\right) \\
= & \int\left(\boldsymbol{e}_{1} \cup \delta \boldsymbol{e}_{2}+\delta \boldsymbol{e}_{2} \cup \boldsymbol{e}_{1}+\boldsymbol{e}_{2} \cup \delta \boldsymbol{e}_{1}+\delta \boldsymbol{e}_{1} \cup \boldsymbol{e}_{2}\right)=0
\end{aligned}
$$

where we have used the property $\int \delta \boldsymbol{e}_{1} \cup_{1} \delta \boldsymbol{e}_{2}=$ $\int\left(\boldsymbol{e}_{1} \cup \delta \boldsymbol{e}_{2}+\delta \boldsymbol{e}_{2} \cup \boldsymbol{e}_{1}\right)$.

To be more precise about the signs in (31) and (32), we give the definition of $S_{\beta}$ for a 2-cochain $\beta \in C^{2}\left(T, \mathbb{Z}_{2}\right)$ :

$$
S_{\beta} S_{\beta^{\prime}}=(-1)^{\int \beta^{\prime} \cup_{1} \beta} S_{\beta+\beta^{\prime}}
$$

or equivalently

$$
S_{\beta}=(-1)^{\sum_{f<f^{\prime} \in \beta} f \cup_{1} f^{\prime}} \prod_{f \in \beta} S_{f}
$$

where we consider the 2-cochain $\beta$ as an ordered set of $f$ with $\beta(f)=1$ and the choice of the order doesn't affect the product due to its property (26). Note that the convention for the product $\prod$ in the ordered set $\left\{f_{1}, f_{2}, \ldots, f_{n}\right\}$ is $\prod_{f \in\left\{f_{1}, f_{2}, \ldots, f_{n}\right\}} S_{f}=S_{f_{n}} \cdots S_{f_{2}} S_{f_{1}}$. We can also define $U_{\beta}$ in the same way. It can be checked that

$$
U_{\beta}=(-1)^{\sum_{f<f^{\prime} \in \beta} f \cup_{1} f^{\prime}} \prod_{f \in \beta} U_{f}=: \prod_{f \in \beta} U_{f}:
$$

where $: \cdots:$ is the normal ordering which places all $X$ operators to the left of $Z$ operators. For example, we have

$$
U_{\delta e}=\left(\prod_{f \supset e} X_{f}\right) \prod_{f^{\prime}} Z_{f^{\prime}}^{\int f^{\prime} \cup_{1} \delta e}
$$

On the other hand, we can show that

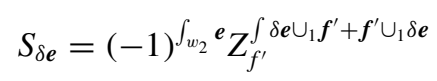

where the 1-chain $w_{2}$ consists of all edges of the triangulation, together with the (02) edge for all " + " tetrahedra and the (13) edge for all "_" tetrahedra:

$$
w_{2}=\sum_{e} e+\sum_{t \in+\text { tetrahedra }} t_{02}+\sum_{t \in-\text { tetrahedra }} t_{13}
$$

This is exactly the 1-chain which is Poincaré dual to the second Stiefel-Whitney class [3]. It is a 1-cycle modulo 2 (that is, its boundary is trivial when regarded as a 0-chain with coefficients in $\mathbb{Z}_{2}$ ). If the topological space corresponding to the triangulation is simply connected, then any 1-cycle is a boundary of some 2-cycle [13]. Thus we can define

$$
S_{\beta}^{E}=(-1)^{\int_{E} \beta} S_{\beta}
$$

where $E$ is a 2 -chain such that $\partial E=w_{2}$. Such a $E$ is not unique, but any two choices differ by a 2-cycle.

One can interpret the 2-chain $E$ as a lattice representation of a spin structure. Indeed, in the context of Riemannian geometry it is well known that the 2nd Stiefel-Whitney class $\boldsymbol{w}_{2}(M) \in H^{2}\left(M, \mathbb{Z}_{2}\right)$ is an obstruction for defining a lift of the structure group of the tangent bundle from $S O(n)$ to $\operatorname{Spin}(n)$. Thus any trivialization of this class leads to a lift of the structure group to $\operatorname{Spin}(n)$ and enables one to define spinors. Since $E$ is a trivialization of the homology 1-cycle Poincaré dual to $\boldsymbol{w}_{2}(M)$, a choice of $E$ is equivalent to a choice of a trivialization of $\boldsymbol{w}_{2}(M)$ and thus can be thought of as implicitly defining a spin structure. It is remarkable that although we are dealing with spinless fermions, a choice of spin structure is still required in order to construct the bosonization map. 
We see that if we identify $S_{f}^{E}$ and $U_{f}$, then the bosonic variables must satisfy a gauge constraint (33). The 3d bosonization map can be summarized as follows:

$$
\begin{aligned}
W_{t} & =\prod_{f \subset t} Z_{f} \longleftrightarrow(-1)^{F_{t}}=-i \gamma_{t} \gamma_{t}^{\prime}, \\
U_{f} & =X_{f}\left(\prod_{f^{\prime}} Z_{f^{\prime}}^{\int f^{\prime} \cup_{1} f}\right) \longleftrightarrow(-1)^{\int_{E} f} S_{f}=(-1)^{\int_{E}} f_{i \gamma_{L(f)}} \gamma_{R(f)}^{\prime}, \\
G_{e} & =\prod_{f \supset e} X_{f}\left(\prod_{f^{\prime}} Z_{f^{\prime}}^{\int \delta e \cup_{1} f^{\prime}}\right) \longleftrightarrow(-1)^{\int_{w_{2}} e} S_{\delta e} \prod_{t}\left[(-1)^{F_{t}}\right]^{\int e \cup_{1} t+t \cup_{1} e}=1,
\end{aligned}
$$

where $w_{2}$ is defined in (40) and $E$ satisfies $\partial E=w_{2}$.

The modified Gauss law looks complicated, but it can be written down more concisely if we describe the spin configurations by a 2 -cochain $B \in C^{2}\left(T, \mathbb{Z}_{2}\right)$. Our convention is that $B(f)=1$ if $Z_{f}=-1$ and $B(f)=0$ if $Z_{f}=1$. Thus the unconstrained Hilbert space is spanned by vectors $|B\rangle$ for all $B$. A 2-form gauge transformation has a 1-cochain $\Lambda$ as a parameter and acts by $B \mapsto B+\delta \Lambda$. For a general $\Lambda$, the Gauss law constraint is given by

$$
\left(\prod_{f \in \delta \Lambda} X_{f}\right)\left(\prod_{f^{\prime}} Z_{f^{\prime}}^{\int \delta \Lambda \cup_{1} f^{\prime}}\right)(-1)^{\int \Lambda \cup \delta \Lambda}=1 .
$$

This formula is proved by $\int \boldsymbol{e} \cup \delta \boldsymbol{e}=0$ and induction:

$$
\begin{gathered}
\left(\prod_{f_{1} \in \delta \Lambda_{1}} X_{f_{1}}\right)\left(\prod_{f_{1}^{\prime}} Z_{f_{1}^{\prime}}^{\delta \Lambda_{1} \cup_{1} f_{1}^{\prime}}\right)(-1)^{\int \Lambda_{1} \cup \delta \Lambda_{1}}\left(\prod_{f_{2} \in \delta \Lambda_{2}} X_{f_{2}}\right)\left(\prod_{f_{2}^{\prime}} Z_{f_{2}^{\prime}}^{\delta \Lambda_{2} \cup_{1} f_{2}^{\prime}}\right)(-1)^{\int \Lambda_{2} \cup \delta \Lambda_{2}} \\
=\left(\prod_{f \in \delta\left(\Lambda_{1}+\Lambda_{2}\right)} X_{f}\right)\left(\prod_{f^{\prime}} Z_{f^{\prime}}^{\delta\left(\Lambda_{1}+\Lambda_{2}\right) \cup_{1} f^{\prime}}\right)(-1)^{\int \Lambda_{1} \cup \delta \Lambda_{1}+\Lambda_{2} \cup \delta \Lambda_{2}}(-1)^{\int \delta \Lambda_{1} \cup{ }_{1} \delta \Lambda_{2}} \\
=\left(\prod_{f \in \delta\left(\Lambda_{1}+\Lambda_{2}\right)} X_{f}\right)\left(\prod_{f^{\prime}} Z_{f^{\prime}}^{\delta\left(\Lambda_{1}+\Lambda_{2}\right) \cup f^{\prime}}\right)(-1)^{\int\left(\Lambda_{1}+\Lambda_{2}\right) \cup \delta\left(\Lambda_{1}+\Lambda_{2}\right)}
\end{gathered}
$$

where we use the identity $\int \delta \Lambda_{1} \cup_{1} \delta \Lambda_{2}=\int \Lambda_{1} \cup \delta \Lambda_{2}+$ $\delta \Lambda_{2} \cup \Lambda_{1}$ in the last equality. Equation (43) can be concisely written as

$$
\left(\prod_{f \in \delta \Lambda} X_{f}\right)(-1)^{\int \Lambda \cup \delta \Lambda+\delta \Lambda \cup_{1} B}=1,
$$

where $B$ is an arbitrary 2-cochain with values in $\mathbb{Z}_{2}$.

Consider now the following 2 -form gauge theory defined on a general triangulated 4D manifold $Y$ :

$$
S(B)=\sum_{t}|\delta B(t)|+i \pi \int_{Y}\left(B \cup B+B \cup_{1} \delta B\right) .
$$

Here $B \in C^{2}\left(Y, \mathbb{Z}_{2}\right)$ is a $\mathbb{Z}_{2}$ field living at each face, and the gauge symmetry acts by $B \rightarrow B+\delta \Lambda$ for an arbitrary 1 -cochain $\Lambda$. The second term is the Steenrod square topological action [14], which is used in Ref. [15] to construct fermionic topological phases. The action is gauge invariant up to a boundary term:

$$
S(B+\delta \Lambda)-S(B)=\int_{\partial Y}\left(\Lambda \cup \delta \Lambda+\delta \Lambda \cup_{1} B\right) .
$$

This boundary term determines the Gauss law for the wave function $\Psi(B)$ on the spatial slice $X=\partial Y$ :

$$
\Psi(B+\delta \Lambda)=(-1)^{\omega(\Lambda, B)} \Psi(B)
$$

where $\omega(\Lambda, B)=\int_{X}\left(\Lambda \cup \delta \Lambda+\delta \Lambda \cup_{1} B\right)$. The Gauss law is the same as the gauge constraint (45). In the following section we use this observation to construct a 4D lattice action for particular Hamiltonian gauge theories with the modified Gauss law.

\section{EUCLIDEAN 3+1D GAUGE THEORIES WITH FERMIONIC DUALS}

In the previous section we constructed a $3 \mathrm{~d}$ bosonization map which works on the kinematic level (that is, is independent of the Hamiltonian). In this section we apply it to some specific models of free fermions and describe the corresponding dual gauge theories. We then construct Euclidean formulations of these gauge theories. We will make use of cup products, and thus will assume that the $3 \mathrm{~d}$ space is triangulated, as in Sec. II C. Accordingly, the 3+1D lattice will be the product of the $3 \mathrm{~d}$ triangulation and discrete time. As explained in the Appendix, (higher) cup products can also be defined on the $3 \mathrm{~d}$ cubic lattice, thus similar considerations can 
be used to find the Euclidean formulation of gauge theories constructed in Sec. II A.

Consider the simplest gauge-invariant Hamiltonian compatible with the modified Gauss law:

$$
H=-A \sum_{f} U_{f}-B \sum_{t} W_{t}
$$

The gauge constraint is

$$
G_{e} \equiv\left(\prod_{f \supset e} X_{f}\right) \prod_{f^{\prime}} Z_{f^{\prime}}^{\int \delta e \cup_{1} f^{\prime}}=1
$$

The partition function can be calculated by transfer matrix method:

$$
Z=\operatorname{Tr} e^{-\beta H}=\operatorname{Tr} T^{M}
$$

where $T$ is the transfer matrix defined as

$$
T=\left(\prod_{e} \delta_{G_{e}, 1}\right) e^{-\delta \tau H}
$$

where $\delta \tau \equiv \beta / M$, and $M \gg 1$ is a large positive integer. The first factor arises from the gauge constraints on the Hilbert space. For calculation purposes, we can rewrite it as

$$
\begin{aligned}
\delta_{G_{e}, 1} & =\frac{1}{2}\left(1+G_{e}\right) \\
& =\frac{1}{2} \sum_{\lambda_{e}= \pm 1}(-1)^{\frac{1-\lambda_{e}}{2} \sum_{f^{\prime} \in \mathrm{NE}(e)} \frac{1-Z_{f^{\prime}}}{2}} \cdot(-1)^{\frac{1-\lambda_{e}}{2} \sum_{f \supset e} \frac{1-X_{f}}{2}}
\end{aligned}
$$

with $\operatorname{NE}(e) \equiv\left\{f \mid \int \delta e \cup_{1} f=1\right\}$. Here $\lambda_{e}$ is the Lagrange multiplier on each edge $e$ of the spatial manifold $M$ and will be consider as $\mathbb{Z}_{2}$ fields living on "temporal" faces later. To calculate the partition function (51), the completed bases are inserted between $T$. Define $|m(\tau)\rangle=\left|\left\{S_{f}\right\}\right\rangle$ as the configuration of spins (in $Z_{f}$ basis). Then the matrix elements of $T$ are

$$
\begin{aligned}
& \left\langle m^{\prime}(\tau+\delta \tau)|T| m(\tau)\right\rangle \\
& \quad=\left\langle m^{\prime}(\tau+\delta \tau)\left|\left(\prod_{v} \delta_{G_{e}, 1}\right) e^{-\delta \tau H}\right| m(\tau)\right\rangle .
\end{aligned}
$$

Next we need to use an identity

$$
\left\langle S^{z^{\prime}}\left|f\left(\sigma^{x}, \sigma^{z}\right)\right| S^{z}\right\rangle=\frac{1}{2} \sum_{S^{x}= \pm 1} f\left(S^{x}, S^{z}\right)(-1)^{\frac{1-S^{x}}{2}\left(\frac{1-S^{z}}{2}+\frac{1-S^{z}}{2}\right)}
$$

where we assume that $\sigma^{x}$ is to the right of $\sigma^{z}$ in the function $f\left(\sigma^{x}, \sigma^{z}\right)$. Plugging this into (54), we get

$$
\begin{aligned}
\left\langle m^{\prime}(\tau+\delta \tau)\right| & \left(\prod_{e} \delta_{G_{e}, 1}\right) e^{-\delta \tau H}|m(\tau)\rangle \\
= & \left\langle m^{\prime}(\tau+\delta \tau)\left|\left(\prod_{e} \delta_{G_{e}, 1}\right)\left(\prod_{f} \sum_{S_{f}^{x}= \pm 1}\left|S_{f}^{x}\right\rangle\left\langle S_{f}^{x}\right|\right) e^{-\delta \tau H}\right| m(\tau)\right\rangle \\
\sim & {\left[\sum_{\lambda_{e}= \pm 1}(-1)^{\frac{1-\lambda e}{2}\left(\sum_{f_{2} \supset e} \frac{1-S_{f_{2}}^{x}}{2}+\sum_{f_{3} \in \mathrm{NE}(e)} \frac{1-S_{f_{3}}^{z}{ }^{\prime}}{2}\right)}(-1)^{\sum_{\lambda_{e}, \lambda_{e^{\prime}}=-1} \int e \cup \delta e^{\prime}}\right] } \\
\times & {\left[\prod_{f} \sum_{S_{f}^{x}= \pm 1}(-1)^{\frac{1-S_{f}^{x}}{2}\left(\frac{1-S_{f}^{z^{\prime}}}{2}+\frac{1-S_{f}^{z}}{2}\right)} e^{A \delta \tau S_{e}^{x} \prod_{f_{1} \in \Delta(f)} S_{f_{1}}^{z}}\right]\left(\prod_{t} e^{B \delta \tau \prod_{f_{4} \subset t} S_{f_{4}}^{z}}\right) }
\end{aligned}
$$

where $\Delta(f) \equiv\left\{f^{\prime} \mid \int \boldsymbol{f}^{\prime} \cup_{1} \boldsymbol{f}=1\right\}$ and the term $(-1)^{\sum_{\lambda_{e}, \lambda_{e^{\prime}}=-1} \int e \cup \delta \boldsymbol{e}^{\prime}}$ comes from pushing all $X_{f}$ operators to the right, which is the same as the last factor of Eq. (43). This term can be expressed as

$$
i \pi \sum_{i} \int a_{i} \cup \delta a_{i}
$$

if we define $a_{i} \in C^{1}\left(M, \mathbb{Z}_{2}\right)$ as 1 -cochain on the spacetial manifold $M$ (ith layer) with $a_{i}(e)=1$ for $\lambda_{e}=-1$ and $a_{i}(e)=0$ for $\lambda_{e}=1$. We can interpret $a_{i}$ at the $i$ th layer as a 2-cochain which lives on the "temporal" faces between the $i$ th and $(i+1)$ th layers.

After extracting this factor, the remaining terms are

$$
\begin{aligned}
& \sum_{\lambda_{e}= \pm 1}\left(\prod_{e}(-1)^{\frac{1-\lambda_{e}}{2} \sum_{f_{3} \subset \mathrm{NE}(e)} \frac{1-S_{f_{3}}^{z}}{2}}\right)\left(\prod_{t} e^{B \delta \tau \prod_{f_{4} \subset t} S_{f_{4}}^{z}}\right)\left[\prod_{f} \sum_{S_{f}^{x}= \pm 1}(-1)^{\frac{1-S_{f}^{x}}{2}\left(\frac{1-S_{f}^{z \prime}}{2}+\frac{1-S_{f}^{z}}{2}+\sum_{e \subset f} \frac{1-\lambda_{e}}{2}\right)} \cdot e^{A \delta \tau S_{e}^{x} \prod_{f_{1} \in \Delta(f)} S_{f_{1}}^{z}}\right] \\
& =\sum_{\lambda_{e}= \pm 1}\left(\prod_{e}(-1)^{\frac{1-\lambda e}{2} \sum_{f_{3} \subset \mathrm{NE}(e)} \frac{1-S_{f_{3}}^{z}}{2}}\right)\left(\prod_{t} e^{B \delta \tau \prod_{f_{4} \subset t} S_{f_{4}}^{z}}\right)\left[\prod_{e}\left(e^{A \delta \tau \prod_{f_{1} \in \Delta(f)} S_{f_{1}}^{z}}+e^{-A \delta \tau \prod_{f_{1} \in \Delta(f)} S_{f_{1}}^{z} S_{f}^{z \prime} S_{f}^{z}} \prod_{e \subset f} \lambda_{e}\right)\right]
\end{aligned}
$$




$$
\begin{aligned}
& \sim \sum_{\lambda_{e}= \pm 1}\left(\prod_{e}(-1)^{\frac{1-\lambda_{e}}{2} \sum_{f_{3} \subset \mathrm{NE}(e)} \frac{1-S_{f_{3}}^{z}}{2}}\right)\left(\prod_{t} e^{B \delta \tau \prod_{f_{4} \subset t} S_{f_{4}}^{z}}\right)\left[\prod_{f} e^{J S_{f}^{z} S_{f}^{z} \prod_{e \subset f} \lambda_{e}}(-1)^{\left(\sum_{f_{1} \in \Delta f f} \frac{1-S_{f_{1}}^{z}}{2}\right)\left(\frac{1-S_{f}^{z}}{2}+\frac{1-S_{f}^{z}}{2}+\sum_{e \subset f} \frac{1-\lambda_{e}}{2}\right)}\right] \\
& =\sum_{\lambda_{e}= \pm 1}(-1)^{\sum_{e}\left(\frac{1-\lambda_{e}}{2} \sum_{f_{3} \mathrm{NE}(e)} \frac{1-S_{f_{3}}^{z}}{2}\right)+\sum_{f}\left(\sum_{f_{1} \in \Delta(f)} \frac{1-S_{f_{1}}^{z}}{2}\right)\left(\frac{1-S_{f}^{z \prime}}{2}+\frac{1-S_{f}^{z}}{2}+\sum_{e_{1} \subset f} \frac{1-\lambda_{e}}{2}\right)} e^{J \sum_{f} S_{f}^{\prime} S_{f}^{z} \prod_{e_{1} \subset f} \lambda_{e_{1}}+B \delta \tau \sum_{t} \prod_{f_{4} \subset t} S_{f_{4}}^{z}}
\end{aligned}
$$

where $\tanh J=e^{-2 A \delta \tau}$. The last line is the usual action for a $4 \mathrm{D} \mathbb{Z}_{2}$ gauge theory except for some sign factors. We regard these factors as coming from a topological action $S_{\text {top }}$. From the penultimate line in (58), we see that $S_{\text {top }}$ contains

$$
i \pi\left[\sum_{e} \frac{1-\lambda_{e}}{2} \sum_{f_{3} \subset \mathrm{NE}(e)} \frac{1-S_{f_{3}}^{z \prime}}{2}+\sum_{f}\left(\sum_{f_{1} \in \Delta(f)} \frac{1-S_{f_{1}}^{z}}{2}\right)\left(\frac{1-S_{f}^{z^{\prime}}}{2}+\frac{1-S_{f}^{z}}{2}+\sum_{e_{1} \subset f} \frac{1-\lambda_{e_{1}}}{2}\right)\right] .
$$

The first term is

$$
\sum_{e} \frac{1-\lambda_{e}}{2} \sum_{f \supset e} \sum_{f \cup_{1} f_{3}=1} \frac{1-S_{f_{3}}^{z \prime}}{2}=\sum_{f}\left(\sum_{e \subset f} \frac{1-\lambda_{e}}{2}\right)\left(\sum_{f \cup_{1} f_{3}=1} \frac{1-S_{f_{3}}^{z \prime}}{2}\right)
$$

which is equal to $\int \delta a_{i} \cup_{1} b_{i+1}$ if we define $b_{i}$ as a 2-cochain on the $i$ th layer with $b_{i}(f)=\frac{1-S_{f}}{2}$. The second term is

$$
\sum_{f}\left(\sum_{f_{1} \mid \int f_{1} \cup_{1} f=1} \frac{1-S_{f_{1}}^{z}}{2}\right)\left(\frac{1-S_{f}^{z \prime}}{2}+\frac{1-S_{f}^{z}}{2}+\sum_{e_{1} \subset f} \frac{1-\lambda_{e_{1}}}{2}\right)
$$

which is $\int b_{i} \cup_{1}\left(b_{i}+b_{i+1}+\delta a_{i}\right)$. Collecting all terms in (57), (60), and (61), we get

$$
S_{\text {top }}\left(\left\{a_{i}\right\},\left\{b_{i}\right\}\right)=i \pi \sum_{i} \int a_{i} \cup \delta a_{i}+\delta a_{i} \cup_{1} b_{i+1}+b_{i} \cup_{1}\left(b_{i}+b_{i+1}+\delta a_{i}\right) .
$$

The usual term $e^{J \sum_{f} S_{f}^{z} S_{f}^{z} \prod_{e_{1} \subset f} \lambda_{e_{1}}+B \delta \tau \sum_{T} \prod_{f_{4} \subset T} S_{f_{4}}^{z}}$ can be written as the exponential of (up to an unimportant constant)

$$
S_{4 \mathrm{D} \text { gauge }}\left(\left\{a_{i}\right\},\left\{b_{i}\right\}\right)=\sum_{i}\left(-2 J \sum_{f}\left|b_{i}(f)+b_{i+1}(f)+\delta a_{i}(f)\right|-2 B \delta \tau \sum_{t}\left|\delta b_{i}(t)\right|\right)
$$

where $|\cdots|$ gives the argument's parity 0 or 1 . Combining (62) and (63), the Euclidean action becomes (up to an additive constant)

$$
S\left(\left\{a_{i}\right\},\left\{b_{i}\right\}\right)=S_{\text {top }}\left(\left\{a_{i}\right\},\left\{b_{i}\right\}\right)+S_{4 \text { Dauge }}\left(\left\{a_{i}\right\},\left\{b_{i}\right\}\right),
$$

which is analogous to action (46) defined by generalized Steenrod square. This action is gauge invariant under gauge transformations

$$
b_{i} \rightarrow b_{i}+\delta \lambda_{i}, a_{i} \rightarrow a_{i}+\delta \mu_{i}+\lambda_{i}+\lambda_{i+1},
$$

where $\lambda_{i}$ are arbitrary 1 -cochains and $\mu_{i}$ are arbitrary 0 -cochains. Indeed, the change in the action is

$$
\begin{aligned}
\frac{\Delta S_{\mathrm{top}}}{(i \pi)}= & \sum_{i} \int\left(a_{i}+\delta \not \lambda_{i}+\lambda_{i}+\lambda_{i+1}\right) \cup\left(\delta \lambda_{i}+\delta \lambda_{i+1}\right) \\
& +\left(\delta \not \mu_{i}+\lambda_{i}+\lambda_{i+1}\right) \cup \delta a_{i}+\left(\delta \lambda_{i}+\delta \lambda_{i+1}\right) \cup \cup_{1} b_{i+1}+\delta a_{i} \cup_{1} \delta \lambda_{i+1} \\
& +\left(\delta \lambda_{i}+\delta \lambda \lambda_{i+1}\right) \cup_{1}^{0} \delta \lambda_{i+1}+\delta \lambda_{i} \cup_{1}\left(b_{i}+b_{i+1}+\delta a_{i}\right) \\
= & \sum_{i} \int a_{i} \cup\left(\delta \lambda_{i}+\delta \lambda_{i+1}\right)+\left(\lambda_{i}+\lambda_{i+1}\right) \cup\left(\delta \lambda_{i}+\delta \lambda_{i+1}\right) \\
& +\left(\lambda_{i}+\lambda_{i+1}\right) \cup \delta a_{i}+a_{i} \cup \delta \lambda_{i+1}+\delta \lambda_{i+1} \cup a_{i}+\lambda_{i} \cup \delta \lambda_{i+1}+\delta \lambda_{i+1} \cup \lambda_{i}+a_{i} \cup \delta \lambda_{i}+\delta \lambda_{i} \cup a_{i} \\
= & \sum_{i} \int \lambda_{i} \cup \delta \lambda_{i}+\lambda_{i+1} \cup \delta \lambda_{i+1}=0
\end{aligned}
$$


where all terms cancel out. In the above computation we assumed periodic time, so that there are no boundary terms. If we do not identify time periodically, the variation is a boundary term

$$
\int\left(\lambda_{0} \cup \lambda_{0}+\delta \lambda_{0} \cup_{1} b_{0}\right)+\left(\lambda_{N} \cup \lambda_{N}+\delta \lambda_{N} \cup_{1} b_{N}\right),
$$

which is the same as the boundary term (47) in the previous section.

We can also check that the action is invariant under a 2form global symmetry

$$
B \rightarrow B+\beta
$$

where a closed 2-cochain $\beta$ can be represented by 2-cochains $\beta_{i}$ (one for each time slice) and 1-cochains $\alpha_{i}$ satisfying $\beta_{i}+$ $\beta_{i+1}+\delta \alpha_{i}=0$. Using a gauge transformation (65) with

$$
\lambda_{i}=\sum_{j=0}^{i-1} \alpha_{j}, \quad \mu_{i}=0
$$

for $i=0,1, \ldots, N-1$, we can see that $\beta_{i}^{\prime}=\beta_{0}$, which is independent of $i$, and $\alpha_{N-1}^{\prime}=\sum_{j=0}^{N-1} \alpha_{j}$ with other $\alpha_{i}^{\prime}=0$. Notice that $\alpha_{N-1}^{\prime}$ is closed since $\beta_{i}^{\prime}=\beta_{i+1}^{\prime}$. Under this 2-form symmetry transformation $\beta^{\prime}$, the action changes by

$$
\begin{aligned}
& \frac{\Delta S_{\mathrm{top}}}{(i \pi)}=\int \underset{\alpha_{N-1}^{\prime} \cup \delta \vec{a}_{N-1}}{ }+\sum_{i} \delta a_{i} \cup_{1} \beta_{0} \\
& +\beta_{0} \cup_{1}\left(\sum_{j} b_{i}+b_{i+1}\right)^{0}+\sum_{i} \beta_{0} \cup_{1} \delta a_{i} \\
& =\sum_{i} \int a_{i} \cup \beta_{0}+\beta_{0} \cup a_{i}+\beta_{0} \cup a_{i}+a_{i} \cup \beta_{0}=0 \text {. }
\end{aligned}
$$

Thus the action is invariant under a global 2-form symmetry, as expected.

\section{GAUGING FERMION PARITY}

We have shown that a lattice fermionic system in $3 \mathrm{~d}$ is dual to a bosonic spin system with the Gauss law constraints. In this section we show how to get rid of the constraints at the expense of coupling fermions to a $\mathbb{Z}_{2}$ gauge field.

Our bosonization map is

$$
\begin{gathered}
-i \gamma_{t} \gamma_{t}^{\prime} \longleftrightarrow W_{t} \equiv \prod_{f \subset t} Z_{f} \\
(-1)^{\int_{E} f}\left(i \gamma_{L(f)} \gamma_{R(f)}^{\prime}\right) \longleftrightarrow U_{f} \equiv X_{f} \prod_{f^{\prime}} Z_{f^{\prime}}^{\int f^{\prime} \cup_{1} f}
\end{gathered}
$$

with gauge constraints

$$
\left(\prod_{f \supset e} X_{f}\right) \prod_{f^{\prime}} Z_{f^{\prime}}^{\int \delta e \cup_{1} f^{\prime}}=1 .
$$

Now, we introduce new $\mathbb{Z}_{2}$ fields (spins), with operators $\tilde{X}, \tilde{Y}$, and $\tilde{Z}$, which live on faces and couple to fermions via a Gauss law constraint

$$
(-1)^{F_{t}}=\prod_{f \subset t} \tilde{Z}_{f}
$$

The fermionic hopping operator must be modified to

$$
S_{f}^{E}=(-1)^{\int_{E} f}\left(i \gamma_{L(f)} \gamma_{R(f)}^{\prime}\right) \tilde{X}_{f}
$$

in order to commute with the Gauss law constraint (73). The bosonization map becomes

$$
\begin{gathered}
-i \gamma_{t} \gamma_{t}^{\prime}=\prod_{f \subset t} \tilde{Z}_{f} \longleftrightarrow W_{t} \equiv \prod_{f \subset t} Z_{f} \\
(-1)^{\int_{E} f}\left(i \gamma_{L(f)} \gamma_{R(f)}^{\prime}\right) \tilde{X}_{f} \longleftrightarrow U_{f} \equiv X_{f} \prod_{f^{\prime}} Z_{f^{\prime}}^{\int f^{\prime} \cup_{1} f}
\end{gathered}
$$

and, similar to (38) and (39), the identification of $U_{\delta e}$ and $S_{\delta e}$ gives

$$
\prod_{f \supset e} \tilde{X}_{f} \longleftrightarrow\left(\prod_{f \supset e} X_{f}\right) \prod_{f^{\prime}} Z_{f^{\prime}}^{\int \delta e \cup_{1} f^{\prime}} .
$$

The Eqs. (75) and (76) define a bosonization map for fermions coupled to a dynamical $\mathbb{Z}_{2}$ gauge field. In this case, there is no constraint on the bosonic variables.

We can apply this modified boson/fermion map to a $\mathbb{Z}_{2}$ version of the Levin-Wen rotor model [4] on general triangulation:

$$
H=-\sum_{t} Q_{t}-\sum_{e} B_{e}
$$

with

$$
\begin{aligned}
& Q_{t}=\prod_{f \subset t} Z_{f} \\
& B_{e}=\prod_{f \supset e}\left(X_{f} \prod_{f^{\prime}} Z_{f^{\prime}}^{\int f \cup_{1} f^{\prime}}\right)=\left(\prod_{f \supset e} X_{f}\right) \prod_{f^{\prime}} Z_{f^{\prime}}^{\int \delta e \cup_{1} f^{\prime}} .
\end{aligned}
$$

Since $Q_{t}$ and $B_{e}$ are just $W_{t}$ and $\left(\prod_{f \supset e} X_{f}\right) \prod_{f^{\prime}} Z_{f^{\prime}}^{\int \delta e \cup_{1} f^{\prime}}$, the above bosonic model is equivalent to a model of a $\mathbb{Z}_{2}$ gauge field coupled to fermions and a Hamiltonian

$$
H=-\sum_{t} \prod_{f \subset t} \tilde{Z}_{f}-\sum_{e} \prod_{f \supset e} \tilde{X}_{f} .
$$

The fermions are static, since the above Hamiltonian does not include fermionic hopping terms. The only interaction between the fermions and the gauge field is via the Gauss law constraint

$$
\prod_{f \subset t} \tilde{Z}_{f}=(-1)^{F_{t}} .
$$

Thus a complicated model bosonic model is mapped to a simple $\mathbb{Z}_{2}$ lattice gauge theory coupled to static fermions.

As another application of the modified bosonization map, consider again the bosonic gauge theory on a cubic lattice with the Hamiltonian (23)

$$
H=-\frac{t}{2} \sum_{i=x, y, z} \sum_{f_{i}} s_{i}\left(L\left(f_{i}\right)\right) U_{f_{i}}\left(1-W_{L\left(f_{i}\right)} W_{R\left(f_{i}\right)}\right)
$$


and a gauge constraint (12). This constrained model is dual to a model of free fermions with Dirac cones. After coupling the fermions to a $\mathbb{Z}_{2}$ gauge field and applying the modified map, we find that the bosonic model (81) without any gauge constraints is equivalent to a fermionic model with the Hamiltonian

$$
\begin{aligned}
H= & -t \sum_{\vec{r}}\left(s_{x}(\vec{r}) \tilde{X}_{x}(\vec{r}) c_{\vec{r}+\hat{x}}^{\dagger} c_{\vec{r}}+s_{y}(\vec{r}) \tilde{X}_{y}(\vec{r}) c_{\vec{r}+\hat{y}}^{\dagger} c_{\vec{r}}\right. \\
& \left.+s_{z}(\vec{r}) \tilde{X}_{z}(\vec{r}) c_{\vec{r}+\hat{z}}^{\dagger} c_{\vec{r}}+\text { H.c. }\right)
\end{aligned}
$$

with $(-1)^{c_{t}^{\dagger} c_{t}}=\prod_{f \subset t} \tilde{Z}_{f}$. The operators $\tilde{W}_{e} \equiv \prod_{f \supset e} \tilde{X}_{f}$ commute with the Hamiltonian, so we can project the Hilbert space into sectors with fixed $\tilde{W}_{e}\left(\tilde{W}_{e}\right.$ is arbitrary \pm 1 as long as it satisfies $\left.\prod_{e \supset v} \tilde{W}_{e}=1\right)$. In the sector $\tilde{W}_{e}=1$ for all $e$, the Hamiltonian (82) returns to (20). The model of unconstrained spins with the Hamiltonian (81) thus can be regarded as a $3 \mathrm{~d}$ analog of Kitaev's honeycomb model.

\section{CONCLUSIONS}

In this paper we constructed a bosonization map for an arbitrary fermionic system on a $3 \mathrm{~d}$ lattice. The lattice can be either a cubic one or a triangulation. The dual bosonic system is a 2-form gauge theory and thus has local constraints (the modified Gauss law). While we did not emphasize this point in the paper, the form of the constraints is largely determined by requiring the system to have a 2 -form $\mathbb{Z}_{2}$ symmetry with a particular't Hooft anomaly. As explained in the end of Sec. 2, another way to understand the constraints is to note that they arise from a 4D 2-form gauge theory with a "topological" term (generalized Steenrod square) in the action

$$
S_{\text {top }}=i \pi \int_{Y} B \cup B+B \cup_{1} \delta B,
$$

where $B$ is a 2 -form $\mathbb{Z}_{2}$ gauge field (i.e., a 2-cochain with values in $\mathbb{Z}_{2}$ ). This action is invariant under a gauge symmetry $B \mapsto B+\delta \lambda$, where $\lambda$ is a 1-cochain, up to a nontrivial boundary term, and it is this boundary term which leads to a modified Gauss law.

One can get rid of the constraint on the bosonic side at the expense of coupling the fermions to a $\mathbb{Z}_{2}$ gauge field (i.e. by gauging the fermion parity). We used this observation to construct a model with spins and no constraints which is dual to a model of free fermions coupled to a static gauge field, and thus is soluble.

The simplest Euclidean 4D 2-form gauge theory which leads to the correct form of the Gauss law for the wave functions has the action (46). It is very likely that this model is dual to a model of free fermions for any triangulated 4D manifold $Y$. It would be very interesting to prove this. Our methods are insufficient here, since they are tied to the Hamiltonian formalism, while (46) makes sense only on a 4D triangulation, but not on a $3 \mathrm{~d}$ triangulation times discrete time, and thus is intrinsically Euclidean. In this paper, instead of attacking this problem head-on, we showed that a complicated-looking 2-form gauge theory with an action (64) is dual to a theory of free fermions. This Euclidean theory leads to the same Gauss law as (46) but has the advantage that it is defined on a $3 \mathrm{~d}$ triangulation times discrete time and thus can be analyzed by our methods.

\section{ACKNOWLEDGMENTS}

Y.-A.C. thanks Djordje Radicevic for many very helpful discussions. This research was supported in part by the US Department of Energy, Office of Science, Office of High Energy Physics, under Award Number DE-SC0011632. A.K. was also supported by the Simons Investigator Award.

\section{APPENDIX A: CHAINS AND COCHAINS NOTATIONS AND (HIGHER) CUP PRODUCTS ON A TRIANGULATION AND A CUBIC LATTICE}

In this section, we review some concepts in algebraic topology and also introduce notations used in this paper. We will always work with an arbitrary triangulation of a simply-connected $n$-dimensional manifold $M_{n}$ equipped with a branching structure (orientations on edges without forming a loop in any triangle). The vertices, edges, faces, and tetrahedra are denoted $v, e, f, t$, respectively. A general $d$-simplex is denoted as $\Delta_{d}$. We can label the vertices of $\Delta_{d}$ as $0,1,2, \ldots, d$ such that the orientations of edges are from the smaller number to the larger number. We denote this $d$-simplex as $\Delta_{d}=\langle 01 \ldots d\rangle$. A finite linear combination of $d$-simplices with coefficients in $\mathbb{Z}_{2}=\{0,1\}$ is called a $d$-chain. $d$-chains form an Abelian group denoted $C_{d}\left(M_{n}, \mathbb{Z}_{2}\right)$. A $\mathbb{Z}_{2}$-valued $d$-chain can be identified with a finite set of $d$ simplices (the set consisting of those simplices whose coefficients are nonzero). A $\mathbb{Z}_{2}$-valued $d$-cochain is a function from the set of $d$-simplices to $\mathbb{Z}_{2}$. The set of all $d$-cochains is an Abelian group denoted $C^{d}\left(M_{n}, \mathbb{Z}_{2}\right)$, For example, a 0-cochain assigns 0 or 1 to all vertices, and a 1-cochain assigns 0 or 1 to all edges. A $d$-cochain can be evaluated on any $d$-chain by evaluating the cochain on each simplex of the $d$-chain and adding up the results modulo 2 .

For every vertex $v$ we define its dual 0 -cochain $v$, which takes value 1 on $v$, and 0 otherwise, i.e., $\boldsymbol{v}\left(v^{\prime}\right)=\delta_{v, v^{\prime}}$. Similarly, e is a 1-cochain $\boldsymbol{e}\left(e^{\prime}\right)=\delta_{e, e^{\prime}}$, and so forth. All dual cochains will be denoted in bold. An evaluation of a cochain $c$ on a chain $c^{\prime}$ will sometimes be denoted $\int_{c^{\prime}} c$. For example, if $c^{\prime}$ is a 2-chain and $c$ is a 2-cochain, $\int_{c^{\prime}} c \equiv c\left(c^{\prime}\right)=\sum_{f \in c^{\prime}} c(f)$. When the integration range is not written, $c$ is assumed to be the top dimension and $\int \boldsymbol{c} \equiv \int_{M_{n}} \boldsymbol{c}$. A $d$-cochain $\boldsymbol{c}_{d} \in$ $C^{d}\left(M_{n}, \mathbb{Z}_{2}\right)$ can be thought of as a collection of $\mathbb{Z}_{2}$-valued variables, one for each $d$-simplex $\Delta_{d}$, whose values given by $\boldsymbol{c}_{d}\left(\Delta_{d}\right)$. We will limit ourselves to the case of $\mathbb{Z}_{2}$-valued cochains, since this is all we need in this paper.

The boundary operator is denoted by $\partial$. For an $n$-simplex $\Delta_{n}, \partial \Delta_{n}$ consists of all boundary $(n-1)$-simplices of $\Delta_{n}$. The coboundary operator is denoted by $\delta$ (not to be confused with the Kronecker delta previously). For example, for a 0cochain $\boldsymbol{v}, \delta \boldsymbol{v}$ is a function on the set of edges which takes value 1 on $e$ if $\partial e$ contains $v$, and takes value 0 otherwise:

$$
\delta \boldsymbol{v}\left(e_{i j}\right)=\boldsymbol{v}\left(\partial e_{i j}\right)=\boldsymbol{v}\left(v_{i}+v_{j}\right)=\delta_{v, v_{i}}+\delta_{v, v_{j}} .
$$

The notation $\Delta_{n}^{1} \supset \Delta_{n^{\prime}}^{2}$ or $\Delta_{n^{\prime}}^{2} \subset \Delta_{n}^{1}$ means that the simplex $\Delta_{n}^{1}$ contains $\Delta_{n^{\prime}}^{2}$ as one of its faces. For example, a simplex $f=\langle 012\rangle$ contains the edges $e=\langle 01\rangle,\langle 02\rangle,\langle 12\rangle \subset f$. 

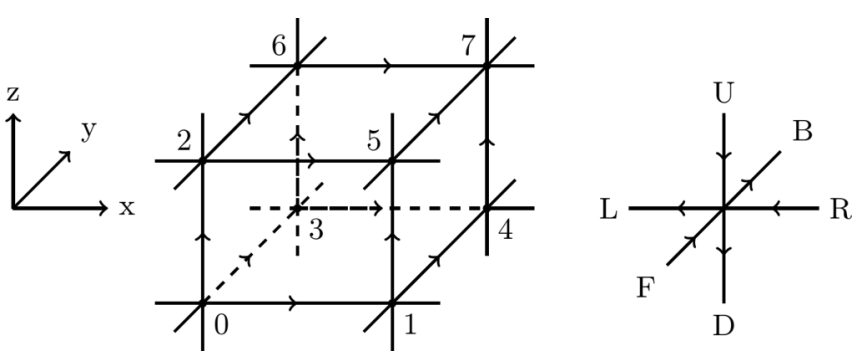

FIG. 5. There are six faces for each cube $c$. U,D,F,B,L,R stand for faces on direction "up," "down," "front," "back," "left," "right." We assign the face U, F, R to be inward and $\mathrm{D}, \mathrm{B}, \mathrm{L}$ to be outward. The $\cup_{1}$ product on two 2-cochain is defined by $\beta \cup_{1} \beta^{\prime}(c)=\beta(L) \beta^{\prime}(B)+\beta(L) \beta^{\prime}(D)+\beta(B) \beta^{\prime}(D)+$ $\beta(U) \beta^{\prime}(F)+\beta(U) \beta^{\prime}(R)+\beta(F) \beta^{\prime}(R)$

In the case of a general triangulation, our bosonization procedure is based on the properties of the cup product $U$ and the higher cup product $\cup_{1}$. These mathematical operations have been defined by Steenrod [14] (see also Appendix B in Ref. [16] for a review) for an arbitrary simplicial complex, but not for a cubic lattice. Below we will define these operations on a triangulation and then describe a version which works for a cubic lattice.

On a lattice triangulation, the cup product $U$ of a $p$-cochain $\alpha_{p}$ and a $q$-cochain $\beta_{q}$ is a $(p+q)$-cochain defined as [17]:

$$
\begin{aligned}
& {\left[\alpha_{p} \cup \beta_{q}\right](\langle 0,1, \ldots, p+q\rangle)} \\
& \quad=\alpha_{p}(\langle 0,1, \ldots, p\rangle) \beta_{q}(\langle p, p+1, \ldots, p+q\rangle) \\
& \quad=\alpha_{p}(0 \sim p) \beta_{q}(p \sim p+q) .
\end{aligned}
$$

The definition of the higher cup product $[3,14]$ is

$$
\begin{aligned}
& {\left[\alpha_{p} \cup_{a} \beta_{q}\right](0,1, \cdots, p+q-a)} \\
& =\sum_{0 \leqslant i_{0}<i_{1}<\cdots<i_{a} \leqslant p+q-a} \alpha_{p}\left(0 \sim i_{0}, i_{1} \sim i_{2}, i_{3} \sim i_{4}, \cdots\right) \\
& \quad \times \beta_{q}\left(i_{0} \sim i_{1}, i_{2} \sim i_{3}, \cdots\right)
\end{aligned}
$$

where $i \sim j$ represents the integers from $i$ to $j$, i.e. $i, i+$ $1, \ldots, j$, and $\left\{i_{0}, i_{1}, \ldots, i_{a}\right\}$ are chosen such that the arguments of $\alpha_{p}$ and $\beta_{q}$ contain $p+1$ and $q+1$ numbers separately. For arbitrary $\mathbb{Z}_{2}$-cochains $A$ and $B$, the cup products satisfy this identity:

$$
A \cup_{a} B+B \cup_{a} A=\delta\left(A \cup_{a+1} B\right)+\delta A \cup_{a+1} B+A \cup_{a+1} \delta B
$$

with $\cup_{0} \equiv \cup$ and $\cup_{-1} \equiv 0$.

To generalize these formulas to the cubic lattice, we first develop an intuition for the cup product $\cup$. On a triangle $\Delta_{012}$, the usual cup product for two 1-cochains $\lambda$ and $\lambda^{\prime}$ is

$$
\lambda \cup \lambda^{\prime}(012)=\lambda(01) \lambda^{\prime}(12) .
$$

We can think of it as starting from vertex 0 , passing through edges 01 and 12 consecutively, and ending at vertex 2 , all the while following the orientation of the edges. Following the same logic, it is intuitive to define the cup product on a square $\square_{0134}$ (the bottom face in Fig. 5) as

$$
\lambda \cup \lambda^{\prime}\left(\square_{0134}\right)=\lambda(01) \lambda^{\prime}(14)+\lambda(03) \lambda^{\prime}(34) .
$$

The two terms come from two oriented paths from vertex 0 to vertex 4 . If $\lambda$ and $\beta$ are a 1-cochain and a 2-cochain, the usual cup product is

$$
\begin{aligned}
& \lambda \cup \beta(0123)=\lambda(01) \beta(123) \\
& \beta \cup \lambda(0123)=\beta(012) \lambda(23) .
\end{aligned}
$$

On the cubic lattice, the corresponding cup products are defined as follows:

$$
\begin{aligned}
\lambda \cup \beta(c)= & \lambda(01) \beta\left(\square_{1457}\right)+\lambda(02) \beta\left(\square_{2567}\right) \\
& +\lambda(03) \beta\left(\square_{3467}\right) \\
\beta \cup \lambda(c)= & \beta\left(\square_{0236}\right) \lambda(67)+\beta\left(\square_{0125}\right) \lambda(57) \\
& +\beta\left(\square_{0134}\right) \lambda(47)
\end{aligned}
$$

where $c$ is a cube whose vertices are labeled in Fig. 5 . For a cup product involving 0 -cochains $\alpha$, the definition is straightforward:

$$
\begin{aligned}
\alpha \cup \beta\left(\square_{0134}\right) & =\alpha(0) \beta\left(\square_{0134}\right) \\
\beta \cup \alpha\left(\square_{0134}\right) & =\beta\left(\square_{0134}\right) \alpha(4) \\
\alpha \cup \lambda(01) & =\alpha(0) \lambda(01) \\
\lambda \cup \alpha(01) & =\lambda(01) \alpha(1) .
\end{aligned}
$$

With the above definitions, it can be checked that the following equalities hold for cubic cochains of degrees 0,1 , and 2 :

$$
\begin{aligned}
\boldsymbol{e}_{1} \cup \delta \boldsymbol{e}_{2} & =\delta \boldsymbol{e}_{1} \cup \boldsymbol{e}_{2}+\delta\left(\boldsymbol{e}_{1} \cup \boldsymbol{e}_{2}\right) \\
\boldsymbol{v} \cup \delta \boldsymbol{f} & =\delta \boldsymbol{v} \cup \boldsymbol{f}+\delta(\boldsymbol{v} \cup \boldsymbol{f}) .
\end{aligned}
$$

The next step is to define the $\cup_{1}$ product on the cubic lattice. It need not satisfy all the properties that $\cup_{1}$ has on a triangulation. The only properties of $\cup_{1}$ that we need are the anticommutativity for faces with the same direction and the identity we used in (34), (44), and (66):

$$
\int \boldsymbol{e}_{1} \cup \delta \boldsymbol{e}_{2}+\delta \boldsymbol{e}_{2} \cup \boldsymbol{e}_{1}=\int \delta \boldsymbol{e}_{1} \cup_{1} \delta \boldsymbol{e}_{2}(\bmod 2) .
$$

Therefore, we only need to define $U_{1}$ product for two 2-cochains so that it satisfies (A10). Our convention for $\cup_{1}$ is shown in Fig. 5:

$$
\begin{aligned}
\beta \cup_{1} \beta^{\prime}(c)= & \beta(L) \beta^{\prime}(B)+\beta(L) \beta^{\prime}(D)+\beta(B) \beta^{\prime}(D) \\
& +\beta(U) \beta^{\prime}(F)+\beta(U) \beta^{\prime}(R)+\beta(F) \beta^{\prime}(R) .
\end{aligned}
$$

Once the $\cup$ and $\cup_{1}$ products are defined on the cubic lattice, the bosonization procedure on a general triangulation can be applied to the cubic lattice. The formalism derived in Sec. II A is just a special case of Sec. II C. In the derivation, (29) and (30) are modified as follows:

$$
\begin{gathered}
S_{\delta e}=(-1)^{\sum_{f<f^{\prime} \in \delta e} f \cup_{1} f^{\prime}} \prod_{f \in \delta e} S_{f} \\
=\prod_{c \mid e \in\{01,14,02,47,67,26\}} W_{c} \\
Z_{f_{1}} Z_{f_{2}} \prod_{f^{\prime} \subset c} Z_{f^{\prime}}^{\left(f_{1}+f_{2}\right) \cup_{1} f^{\prime}+f^{\prime} \cup_{1}\left(f_{1}+f_{2}\right)} \\
= \begin{cases}W_{c}, & \text { if } e \in\{01,14,02,47,67,26\} \\
0, & \text { otherwise }\end{cases}
\end{gathered}
$$



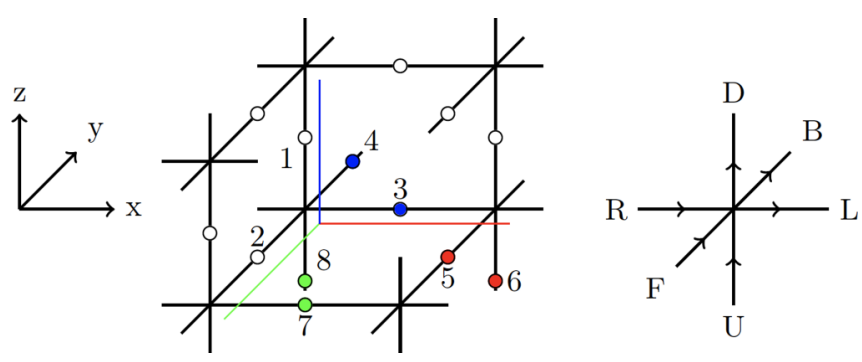

FIG. 6. We rotate the axis $\mathrm{U}, \mathrm{D}, \mathrm{F}, \mathrm{B}, \mathrm{R}, \mathrm{L}$ in Fig. 5 to match the result in Fig. 2. Notice that the cube above is dual lattice and edges $1,2,3 \ldots$ in the dual lattice represent faces in the original lattice.

for faces $f_{1}$ and $f_{2}$ join at the edge $e$. We implicitly choose $w_{2}=0$ in (39). We can use the $\cup_{1}$ product defined above to reproduce the fermionic hopping terms defined by framing in Fig. 2. The hopping term defined by Eq. (27) is

$$
U_{f}=X_{f} \prod_{f^{\prime}} Z_{f^{\prime}}^{\int f^{\prime} \cup_{1} f} .
$$

Figure 6 is dual to Fig. 5. Therefore, faces in Fig. 5 become edges in Fig. 6. Consider the hopping term along dual edge 3. On the dual vertex to the right, it represents the face R. From terms $\beta(F) \beta^{\prime}(R)$ and $\beta(U) \beta^{\prime}(R)$, the hopping term contains $Z_{5}$ (from $\mathrm{F}$ ) and $Z_{6}$ (from $\mathrm{U}$ ). On the dual vertex to the left, it represents the face L. Since there is no $\beta(D) \beta^{\prime}(L)$ or $\beta(B) \beta^{\prime}(L)$ term, it contributes nothing. So we have

$$
U_{3}=X_{3} Z_{5} Z_{6}
$$

Similarly, for edge 2 , the hopping term has $Z_{7}$ [from $\left.\beta(L) \beta^{\prime}(B)\right]$ and $Z_{8}$ [from $\beta(U) \beta^{\prime}(F)$ ]

$$
U_{2}=X_{2} Z_{7} Z_{8}
$$

For edge 1 , the hopping term has $Z_{3}$ [from $\left.\beta(L) \beta^{\prime}(D)\right]$ and $Z_{4}$ [from $\beta(B) \beta^{\prime}(D)$ ]

$$
U_{1}=X_{1} Z_{3} Z_{4}
$$

We get the exact same hopping terms defined by "framing" in Fig. 2. We can interpret the choice of framing as a definition of $\cup_{1}$ product on the cubic lattice.

\section{APPENDIX B: REVIEW OF ORDINARY AND MODIFIED $\mathbb{Z}_{2}$ LATTICE GAUGE THEORIES}

In this Appendix we compare the Hamiltonian formulation of ordinary and modified $\mathbb{Z}_{2}$ lattice gauge theories. A very general definition of a $\mathbb{Z}_{2}$ lattice gauge theory involves a collection of qubits (spins), with physical states required to satisfy commuting constraints labeled by a set $S$. Thus the Hilbert space is

$$
\left.\mathcal{H}=\left\{|\Psi\rangle \in \bigotimes \mathbb{C}^{2}\left|G_{i}\right| \Psi\right\rangle=|\Psi\rangle \forall i \in S\right\}
$$

where $\left\{G_{i}\right\}_{i \in S}$ is a set of commuting operators each of which squares to 1 . Most often, one considers gauge theories where qubits live on edges of a lattice. We will refer to such gauge theories as 1-form gauge theories. Two states of the qubit living on an edge $e$ can be parameterized by a classical variable $a_{e} \in\{0,1\}$. The Pauli operators act as follows:

$$
\begin{aligned}
X_{e}\left|a_{e}\right\rangle & =\left|a_{e}+1(\bmod 2)\right\rangle \\
Z_{e}\left|a_{e}\right\rangle & =(-1)^{a_{e}}\left|a_{e}\right\rangle .
\end{aligned}
$$

For 1-form gauge theories, the set $S$ is the space of all vertices. In the case of ordinary 1-form gauge theories, the constraint corresponding to the vertex $v$ is

$$
G_{v}^{0}=\prod_{e \supset v} X_{e}
$$

Each configuration of the classical variables $\left\{a_{e}\right\}$ is a basis vector in the unconstrained Hilbert space. It can also be though of as a 1 -cochain $a \in C^{1}\left(M, \mathbb{Z}_{2}\right)$ on the spatial manifold $M$. Imposing the constraint $G_{v}^{0}|\Psi\rangle=|\Psi\rangle$ is equivalent to requiring physical states to be invariant under the transformation

$$
\boldsymbol{a} \mapsto \boldsymbol{a}+\delta \boldsymbol{v} .
$$

Thus a physical state must be invariant under a transformation $\boldsymbol{a} \mapsto \boldsymbol{a}+\delta \boldsymbol{\phi}$, where $\boldsymbol{\phi}$ is an arbitrary 0-cochain. This is the reason to call this model a $\mathbb{Z}_{2}$ gauge theory.

Physical observables must commute with all constraints. In other words, they must be gauge invariant. The algebra of gauge-invariant local observables for an ordinary 1-form gauge theory has generators

$$
X_{e}, W_{f} \equiv \prod_{e \subset f} Z_{e}
$$

where the latter one is often referred as the "flux" or "field strength."

Let us now specialize to the case of 1-form gauge theory in two spatial dimensions (with the constraints as above). One can show that this theory is dual to a model of spins living on faces of the $2 \mathrm{~d}$ lattice. The Hilbert space of this theory is unconstrained. If we denote by $X_{f}$ and $Z_{f}$ the Pauli matrices generating the algebra of observables on the face $f$, the duality map is

$$
\begin{aligned}
& W_{f}=\prod_{e \subset f} Z_{e} \longleftrightarrow Z_{f}, \\
& X_{e} \longleftrightarrow X_{L(e)} X_{R(e)}, \\
& G_{v}^{0}=\prod_{e \supset v} X_{e} \longleftrightarrow \prod_{e \supset v}\left(X_{L(e)} X_{R(e)}\right)=1,
\end{aligned}
$$

where $L(e)$ and $R(e)$ are the two faces adjacent to the edge $e$. This map identifies the algebra of gauge-invariant local observables for the ordinary 1 -form $\mathbb{Z}_{2}$ gauge theory in $2 \mathrm{~d}$ with the algebra of those local observables in the spin model which commute with the net spin-parity operator $\prod_{f} Z_{f}$. Note that the constraint operator $G_{v}^{0}$ maps to the identity operator. This agrees with the fact that the Hilbert space of the dual spin model is unconstrained.

On a $2 \mathrm{~d}$ square lattice, our modified $\mathbb{Z}_{2}$ gauge theory has the same variables, but the constraint corresponding to a vertex $v$ is now:

$$
G_{v} \equiv W_{\mathrm{NE}(v)} \prod_{e \supset v} X_{e}=1
$$


where $\operatorname{NE}(v)$ is the face northeast of $v$. The algebra of gauge-invariant local observables (that is, the algebra of local observables commuting with all constraints) is generated by

$$
U_{e}, W_{f}=\prod_{e \subset f} Z_{e},
$$

where $U_{e}$ is defined in (3). The unconstrained Hilbert space is spanned by vectors $|\boldsymbol{a}\rangle$, where $\boldsymbol{a} \in C^{1}\left(M, \mathbb{Z}_{2}\right)$. The constraint $G_{v}$ acts as follows:

$$
G_{v}:|\boldsymbol{a}\rangle \mapsto(-1)^{\sum_{e \subset N E(v)} a_{e}}|\boldsymbol{a}+\delta \boldsymbol{v}\rangle .
$$

This modified $\mathbb{Z}_{2}$ gauge theory is dual to a model of fermions via the map (5).

In the $3 \mathrm{~d}$ case, the relevant gauge theory is a 2 -form $\mathbb{Z}_{2}$ gauge theory. That is, qubits live on faces, while the set $S$ labeling the constraints is the set of all edges. We denote by $X_{f}$ and $Z_{f}$ the Pauli operators acting on the qubit on a face $f$. The ordinary gauge constraint corresponding to the edge $e$ reads

$$
G_{e}^{0}=\prod_{f \supset e} X_{f}
$$

The algebra of gauge-invariant local observables (that is, local observables commuting with all the constraints) is generated by

$$
X_{f}, W_{t} \equiv \prod_{f \subset t} Z_{f} .
$$

Before one imposes the constraints, the Hilbert space is spanned by vectors $|\boldsymbol{b}\rangle$, where $\boldsymbol{b} \in C^{2}\left(M, \mathbb{Z}_{2}\right)$. The value of the 2-cochain $\boldsymbol{b}$ on a face $f$ encodes one of the two states of the qubit on $f$. The constraint $G_{e}^{0}$ acts on these basis vectors by

$$
G_{e}^{0}:|\boldsymbol{b}\rangle \mapsto|\boldsymbol{b}+\delta \boldsymbol{e}\rangle .
$$

Thus physical states are those which are invariant under $\boldsymbol{b} \mapsto$ $\boldsymbol{b}+\delta \boldsymbol{\lambda}$ for all 1 -cochains $\lambda$. This ordinary 2 -form $\mathbb{Z}_{2}$ gauge theory is dual to a model of spins living at tetrahedrons (generated by $X_{t}$ and $Z_{t}$ ):

$$
\begin{aligned}
& W_{t}=\prod_{f \subset t} Z_{e} \longleftrightarrow Z_{t}, \\
& X_{f} \longleftrightarrow X_{L(f)} X_{R(f)}, \\
& G_{e}^{0}=\prod_{f \supset e} X_{f} \longleftrightarrow \prod_{f \supset e}\left(X_{L(f)} X_{R(f)}\right)=1,
\end{aligned}
$$

where $L(f)$ and $R(f)$ are the two tetrahedrons adjacent to the face $f$.

In our modified 2 -form $\mathbb{Z}_{2}$ gauge theory on a $3 \mathrm{~d}$ triangulation, we change the gauge constraints to

$$
G_{e}=\prod_{f \supset e} X_{f}\left(\prod_{f^{\prime}} Z_{f^{\prime}}^{\int \delta e \cup_{1} f^{\prime}}\right) .
$$

The algebra of gauge-invariant local observables is generated by:

$$
U_{f}=X_{f}\left(\prod_{f^{\prime}} Z_{f^{\prime}}^{\int f^{\prime} \cup_{1} f}\right), \quad W_{t}=\prod_{f \subset t} Z_{f} .
$$

The unconstrained Hilbert space is spanned by vectors $|\boldsymbol{b}\rangle$, where $\boldsymbol{b} \in C^{2}\left(M, \mathbb{Z}_{2}\right)$. The constraint operators act as follows:

$$
G_{e}:|\boldsymbol{b}\rangle \mapsto(-1)^{\int \delta \boldsymbol{e} \cup_{1} \boldsymbol{b}}|\boldsymbol{b}+\delta \boldsymbol{e}\rangle .
$$

One of the main results of this paper is that the modified 2 -form $\mathbb{Z}_{2}$ gauge theory is dual to a theory of fermions via the map (42).
[1] Y.-A. Chen, A. Kapustin, and D. Radicevic, Exact bosonization in two spatial dimensions and a new class of lattice gauge theories, Ann. Phys. 393, 234 (2018).

[2] In contrast, a 2-form $\mathbb{Z}_{2}$ gauge theory with the standard Gauss law is mapped, by a $3 \mathrm{~d}$ version of the Kramers-Wannier duality, to a theory of bosonic spins.

[3] D. Gaiotto and A. Kapustin, Spin TQFTs and fermionic phases of matter, Int. J. Mod. Phys. A 31, 1645044 (2016).

[4] M. Levin and X.-G. Wen, Quantum ether: Photons and electrons from a rotor model, Phys. Rev. B 73, 035122 (2006).

[5] E. Fradkin, Jordan-Wigner Transformation for Quantum Spin Systems in Two Dimensions and Fractional Statistics, Phys. Rev. Lett. 63, 322 (1989).

[6] S. B. Bravyi and A. Y. Kitaev, Fermionic quantum computation, Ann. Phys. 298, 210 (2002).

[7] R. C. Ball, Fermions without Fermion Fields, Phys. Rev. Lett. 95, 176407 (2005).

[8] F. Verstraete and J. I. Cirac, Mapping local hamiltonians of fermions to local hamiltonians of spins, J. Stat. Mech: Theory Exp. (2005) P09012.
[9] S. Mandal and N. Surendran, Exactly solvable kitaev model in three dimensions, Phys. Rev. B 79, 024426 (2009).

[10] S. Ryu, Three-dimensional topological phase on the diamond lattice, Phys. Rev. B 79, 075124 (2009).

[11] There are many choices of framing and accordingly many versions of the bosonization map. By construction, they are related by automorphisms of the algebra of observables.

[12] M. Creutz, Emergent spin, Ann. Phys. 342, 21 (2014).

[13] Actually, one can show that the second Stiefel-Whitney class of any oriented 3-manifold is trivial, and therefore the above 1cycle is a boundary even in the non-simply-connected case. Our bosonization procedure works also for non-simply-connected spaces. The only difference is that apart from local Gauss law constraints one also needs to impose nonlocal constraints, one for each nontrivial class in $H_{1}\left(X, \mathbb{Z}_{2}\right)$. For example, for a 3 -torus one would need to impose three constraints, one for each direction $x, y, z$. These constraints on the bosonic side are needed to reproduce the freedom to impose either periodic or antiperiodic boundary conditions on the fermions. But in 
this paper we limit ourselves to topologically-trivial (simplyconnected) spaces.

[14] N. E. Steenrod, Products of cocycles and extensions of mappings, Ann. Math. 48, 290 (1947).

[15] T. Lan, C. Zhu, and X.-G. Wen, Fermion decoration construction of symmetry protected trivial orders for fermion systems with any symmetries $G_{f}$ and in any dimensions, arXiv: 1809.01112.

[16] A. Kapustin and N. Seiberg, Coupling a QFT to a TQFT and duality, J. High Energy Phys. 04 (2014) 001.

[17] A. Hatcher, Algebraic Topology (Cambridge Univ. Press, Cambridge, 2000). 